\title{
Rôle de l'endocytose dans la protéolyse de l'insuline et du glucagon dans l'hépatocyte
}

L'insuline et le glucagon, hormones toutes deux pancréatiques, contrôlent la glycémie en occupant des récepteurs spécifiques localisés à la surface de l'hépatocyte et en produisant des signaux biochimiques antagonistes. Les complexes hormone/récepteur sont internalisés et s'accumulent dans le compartiment endosomique de l'hépatocyte. Alors que le récepteur de l'insuline accède à l'endosome sous une forme active, le récepteur du glucagon internalisé est découplé de son système de transduction, qui reste à la surface cellulaire, et perd sa capacité à engendrer son second messager (l'AMPc). Dans les endosomes, les deux ligands sont dégradés selon un processus dépendant de l'ATP par différentes thiolendopeptidases présentant une activité optimale à $\mathrm{pH}$ acide et localisées dans la partie luminale des vésicules. La dissociation des complexes hormone/récepteur, requise pour une dégradation des ligands, est facilitée par l'acidité des endosomes. Celle-ci est maintenue par l'activité de pompes à protons dépendantes de l'ATP. La protéase responsable de la dégradation endosomique de l'insuline a été récemment partiellement purifiée et n'est ni structurellement ni immunologiquement reliée à l'insulinase cytosolique, une thiol-métalloendopeptidase proposée antérieurement comme l'enzyme clé du métabolisme

François Authier Barry I. Posner John J.M. Bergeron

\footnotetext{
ADRESSE

F. Authier : chercheur post-doctoral. B. I. Posner: professeur de médecine. J. J. M. Bergeron: professeur d'anatomie et de biologie cellulaire. Départements de médecine et d'anatomie-biologie cellulaire de l'université de McGill, Montréal, H3A 2B2, Québec, Canada.
} de l'insuline. En réalité, l'insulinase cytosolique vient d'être identifiée comme la première protéase associée au peroxysome, et son rôle physiologique dans ce compartiment reste encore incertain à ce jour.

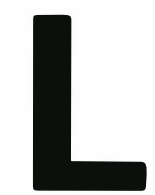

es hormones pancréatiques insuline et glucagon sont sécrétées respectivement par les cellules $\beta$ et $\alpha$ des îlots de Langerhans pour être ensuite présentées au foie par la veine porte. Les clairances métaboliques de l'insuline et du gluca- gon (c'est-à-dire le volume plasmatique débarrassé de ces hormones par unité de temps) déterminées chez le chien sont respectivement de 19,5 et $13 \mathrm{ml} \cdot \mathrm{kg}^{-1} \cdot \mathrm{min}^{-1}$ avec des demi-vies respectives de 4 et 5,5 min, indiquant que ces deux hormones sont rapidement métabolisées par 
l'organisme après leur sécrétion pancréatique [1]. La contribution du foie dans la clairance métabolique totale de ces deux hormones est d'environ $40 \%$, démontrant que le tissu hépatique joue un rôle prépondérant dans le métabolisme physiologique de ces deux polypeptides. Ainsi, en un seul passage transhépatique, plus de $40 \%$ de ces deux hormones sont extraits de la circulation portale consécutivement à leur liaison sur des récepteurs spécifiques situés en majorité à la surface de l'hépatocyte, leur cellule cible [2]. Dès 1975, Terris et Steiner [3] ont défini l'importance de la liaison de l'insuline sur son récepteur et de son internalisation dans l'hépatocyte. Ces auteurs ont montré une dégradation de l'insuline par des hépatocytes isolés et par le foie isolé perfusé. En outre, ils ont suggéré que cette dégradation requière la liaison préalable de l'hormone sur un récepteur, à la différence de la dégradation de l'insuline par les membranes plasmiques isolées [3].
Le métabolisme hépatique des hormones pancréatiques est un processus physiologique important pour la terminaison d'un signal hormonal, pour le maintien d'un taux approprié d'effecteur dans la circulation sanguine et pour l'éventuelle génération de fragments biologiquement actifs à partir de la molécule native. Pour ces raisons, la nature et la localisation subcellulaire des protéases responsables de la dégradation de l'insuline et du glucagon ont été particulièrement étudiées dans ce type cellulaire. Au cours des deux dernières décennies, il était généralement admis que le lysosome était l'organite intracellulaire impliqué dans l'accumulation et la dégradation d'un grand nombre de macromolécules internalisées par l'hépatocyte. Ce n'est que récemment qu'a été établi le rôle des vésicules intracellulaires impliquées au stade précoce de l'endocytose, ou endosomes, dans la dégradation intrahépatocytaire de l'insuline et du glucagon [4].

\section{L'endosome: site majeur d'accumulation de l'insuline et du glucagon internalisés}

La première étape du mécanisme d'action et de dégradation de l'insuline et du glucagon est commune: c'est la liaison à des récepteurs spécifiques localisés dans la membrane plasmique et couplés à deux systèmes de transduction transmembranaire distincts. Pour le glucagon, l'une des conséquences de sa liaison aux récepteurs est une stimulation immédiate de l'adénylyl cyclase via une protéine-G stimulatrice (protéine liant les nucléotides guanyliques comme le GTP), entrainant ainsi une élévation du taux d'AMPc intracellulaire (figure 1). En ce qui concerne l'insuline, sa liaison à la sous-unité $\alpha$ du récepteur induit un changement conformationnel du récepteur provoquant son autophosphorylation sur des résidus sérine et tyrosine ainsi que la stimulation

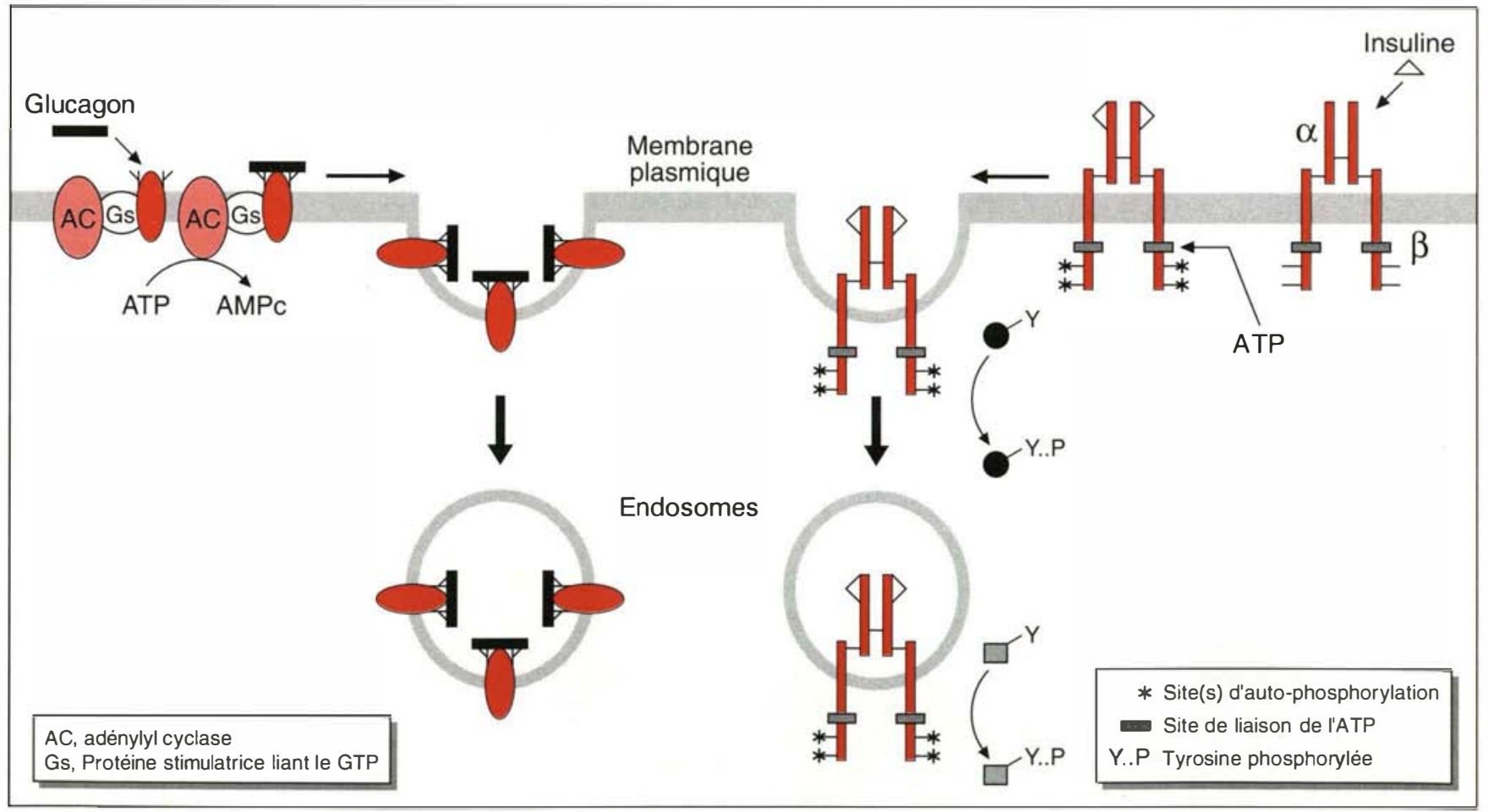

Figure 1. Devenir de l'insuline et du glucagon dans l'hépatocyte. Après liaison de l'insuline et du glucagon à leur récepteur respectif, les complexes hormone/récepteur sont internalisés et s'accumulent dans le compartiment endosomique. Alors que l'activité fonctionnelle du récepteur de l'insuline est maintenue dans ce compartiment, le récepteur du glucagon accède à l'endosome sous une forme inactive, son système de transduction du signal restant à la surface cellulaire. La transduction du signal insulinique est schématisé par la phosphorylation sur des tyrosines de substrats endogènes (cercle et carré).

$\mathrm{m} / \mathrm{s} n^{\circ} 2$ vol. 10 , féunier 94 


\section{RÉFÉRENCES}

1. Jaspan JB, Polonsky KS, Lewis M, Pensler J, Pugh W, Moossa AR, Rubenstein AH. Hepatic metabolism of glucagon in the dog: contribution of the liver to overall metabolic disposal of glucagon. Am J Physiol $1981 ; 240$ : 233-44.

2. Hagopian WA, Tager HS. Hepatic glucagon metabolism. Correlation of hormone processing by isolated canine hepatocytes with glucagon metabolism in man and in the dog. J Clin Invest 1987; 79: 409-17.

3. Terris S, Steiner D. Binding and degradation of ${ }^{125}$ I-insulin by rat hepatocytes. J Biol Chem 1975; 250 : 8389-98.

4. Authier F, Posner BI, Bergeron IJM. Hepatic endosomes are a major physiological locus of insulin and glucagon degradation in vivo. In : Schwartz AL, Ciechanover A, eds. Modern cell biology. New York Wiley-Iiss, 1994 (sous presse).

5. Blache $\mathrm{P}$, Kervran $\mathrm{A}$, Dufour $\mathrm{M}$, et al Glucagon-(19-29), a Ca ${ }^{2+}$ pump inhibitory peptide, is processed from glucagon in the rat liver plasma membrane by a thiol endopeptidase. J Biol Chem 1990 ; 265 : 21514-9.

6. Sheetz MJ, Tager HS. Characterization of a glucagon receptor-linked protease from canine hepatic plasma membranes. Partial purification, kinetic analysis and determination of sites for hormone processing. J Biol Chem 1988; 263: 19210-7.

7. Bergeron IJM, Sikstrom R, Hand AR, Posner BI. Binding and uptake of ${ }^{125} \mathrm{I}-$ insulin into rat liver hepatocytes and endothelium: an in vivo radioautographic study. J Cell Biol 1979; 80 : 427-43.

8. Watanabe J, Kanamura S, Asada-Kubota M, Kanai K, Oka M. Receptor-mediated endocytosis of glucagon in isolated mouse hepatocytes. Anat Rec 1984; 210 : 557-67.

9. Posner BI, Patel BA, Khan MN, Bergeron IJM. Effect of chloroquine on the internalization of ${ }^{125}$ I-insulin into subcellular fractions of rat liver. Evidence for an effect of chloroquine on Golgi elements. J Biol Chem 1982; 257: 5789-99.

10. Kay DG, Khan MN, Posner BI, Berge ron JJM. In vivo uptake of insulin into hepatic Golgi fractions : application of the diaminobenzidine-shift protocol. Biochem Biophys Res Commun 1984; 123 : 1144-8.

11. Authier F, Janicot M, I.ederer F, Desbuquois B. Fate of injected glucagon taken up by rat liver in vivo. Biochem J $1990 ; 272$ : 703-12.

12. Bergeron JJM, Cruz J, Khan MN, Posner BI. Uptake of insulin and other ligands into receptor-rich endocytic components of target cells: the endosomal apparatus. Annu Rev Physiol 1985; 47 : 383-403.

13. De Diego JG, Gorden P, Carpentier JI. The relationship of ligand receptor mobility to internalization of polypeptide hormones and growth factors. Endocrinology $1991 ; 128: 2136-40$ d'une activité kinase, activités toutes deux situées dans le domaine cytosolique des chaînes $\beta$ du récepteur (figure 1).

Parallèlement à la génération des signaux biochimiques induite par l'occupation des récepteurs, des études menées avec des hépatocytes intacts et à l'aide de systèmes acellulaires sur membranes plasmiques isolées ont montré que l'insuline et le glucagon, associés à la membrane plasmique, sont partiellement dégradés par des activités protéolytiques actives à $\mathrm{pH}$ neutre [4]. Alors que les protéases responsables de la dégradation de l'insuline à ce niveau n'ont pas encore été caractérisées, au moins trois activités protéolytiques membranaires semblent impliquées dans la dégradation du glucagon: une aminopeptidase qui clive les liaisons Gln ${ }^{3}-G_{y}{ }^{4}$ et $\mathrm{Phe}^{6}-\mathrm{Thr}^{7}$ et engendre les fragments 4-29 et 7-29 qui sont libérés dans la circulation sus-hépatique [2] ; une thiol-endopeptidase qui clive la liaison $\mathrm{Arg}^{18}-\mathrm{Ala}^{19}$ et engendre le fragment 19-29 qui module sélectivement l'activité de la pompe à $\mathrm{Ca}^{2+}$ dépendante de l'ATP de la membrane plasmique [5]; et une sérine protéase, fonctionnellement liée au récepteur, dont le clivage majoritaire se situe au niveau de la liaison $T_{y r}{ }^{13}$-Leu ${ }^{14}$ et qui engendre le fragment 1-13 restant associé au récepteur [6].

L'événement morphologique e t biochimique majeur qui caractérise le devenir de ces deux hormones associées à la surface cellulaire est l'internalisation des complexes ligand/récepteur. A l'origine, des études de microscopie électronique couplées à l'autoradiographie ont montré que la disparition des deux hormones de la surface cellulaire s'accompagne de leur accumulation dans un compartiment intracellulaire $[7,8]$. En outre, il fut montré morphologiquement que ces vésicules d'endocytose se distinguaient du compartiment lysosomial caractérisé par des mesures cytochimiques de la phosphatase acide, une enzyme marqueur du lysosome [7]. Dans ces mêmes études, moins de $5 \%$ de l'insuline radioactive étaient retrouvés dans les lysosomes secondaires exprimant l'activité phosphatase acide, suggérant déjà que le lysosome n'était pas un organite majeur d'accumulation de l'insuline internalisée [7, 9].

Des études de fractionnement subcellulaire ont ensuite confirmé et étendu ces études morphologiques. L'étude du devenir subcellulaire de l'insuline et du glucagon iodés, captés par le foie de rat in vivo, ont montré une association séquentielle des deux ligands aux membranes plasmiques aux temps précoces, puis à des organites de faible densité à des temps plus tardifs coïncidant avec l'activité galactosyltransférase, une enzyme marqueur du Golgi [7, 9]. L'emploi de la méthode de déplacement de densité des organites endocytiques par la diaminobenzidine a clairement démontré que les structures accumulant l'insuline et le glucagon étaient distinctes de l'activité galactosyltransférase, excluant donc leur appartenance aux saccules golgiennes [10, 11]. En outre, très peu de radioactivité était retrouvée dans les fractions lysosomiales, confirmant donc que les deux ligands étaient internalisés dans des structures endocytiques physiquement distinctes, à la fois, des éléments de Golgi et des lysosomes [11, 12]. Malgré un cheminement intracellulaire commun de l'insuline et du glucagon dans l'hépatocyte, des différences existent cependant dans leur vitesse et leur taux d'internalisation. En fait, la vitesse d'association au compartiment endosomique est plus rapide pour l'insuline (1 à $2 \mathrm{~min}$ ) que pour le glucagon (20 min), avec un taux d'association environ dix fois plus important pour l'insuline. Des études morphologiques de microscopie électronique couplée à l'autoradiographie ont confirmé que la cinétique et le taux d'internalisation des hormones insuline et glucagon et du facteur de croissance épidermique (EGF) étaient différents dans les hépatocytes isolés [13]. L’internalisation est très rapide pour l'EGF (50\% de l'EGF est internalisé en $10 \mathrm{~min})$, rapide pour l'insuline (40\% de l'insuline est internalisée en $10 \mathrm{~min}$ ) et lente pour le glucagon $(25 \%$ du glucagon est internalisé en $30 \mathrm{~min})$. Ces différences découlent d'une mobilité moindre des complexes glucagon/récepteur 


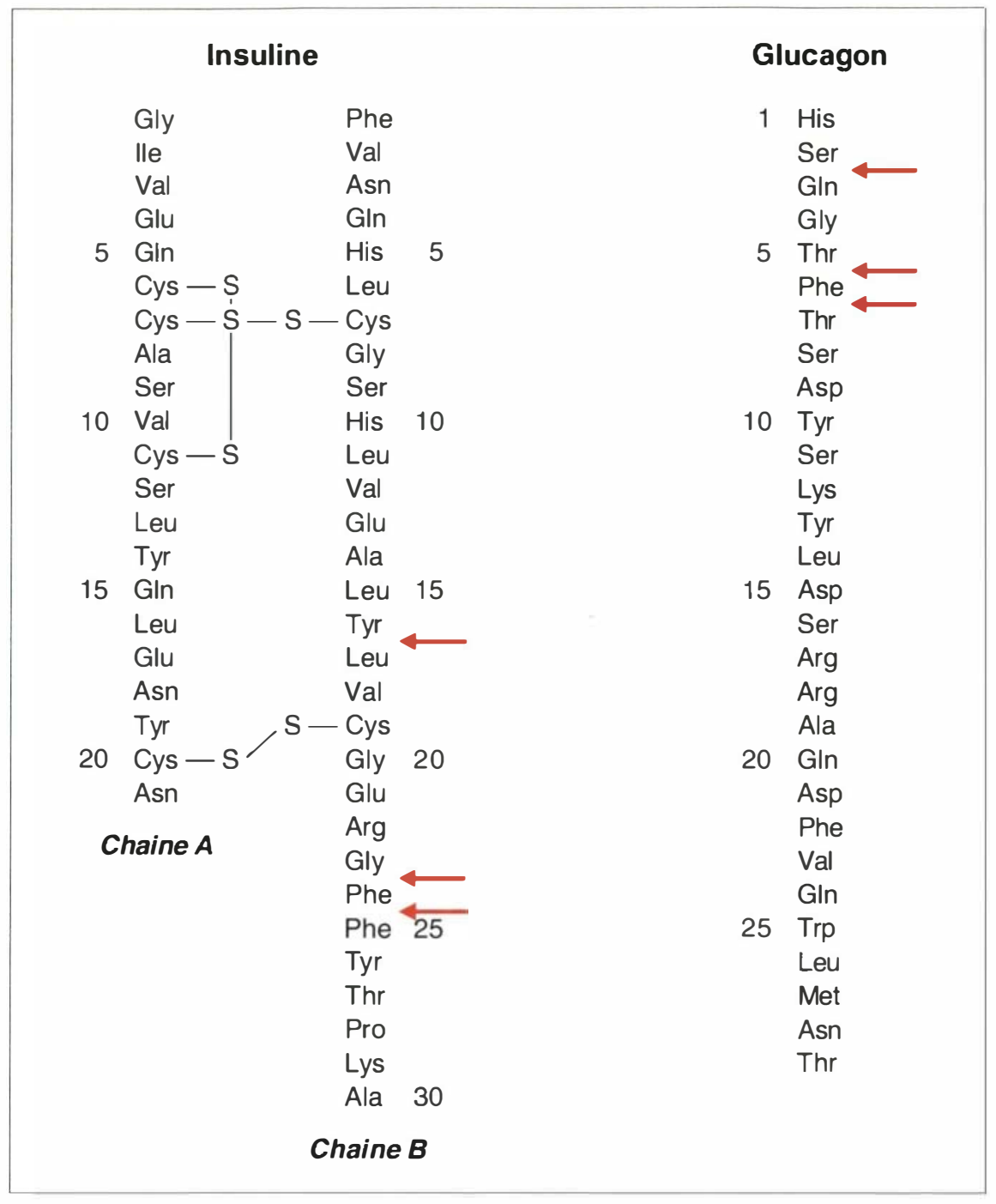

Figure 2. Sites des clivage protéolytiques de l'insuline et du glucagon dans l'endosome. Les flèches rouges indiquent les clivages peptidiques identifiés dans les molécules radioactives d'insuline [19] et de glucagon [11, 21]. IIs ont été déterminés par une purification par chromatographie liquide haute performance en phase inversée des produits de dégradation radioactifs engendrés dans l'endosome, suivie d'une analyse par radioséquençage des liaisons peptidiques clivées.

dans le plan de la membrane plasmique relativement aux autres complexes insuline/récepteur et EGF/récepteur, et pourraient trouver leur origine dans le nombre élevé de domaines transmembranaires du récepteur du glucagon (sept domaines comme tous les récepteurs couplés à l'adénylate cyclase [14]) comparativement à ceux, plus réduits, des récepteurs de l'insuline (deux domaines) et de l'EGF (un domaine) [13].

Les études portant sur le devenir des récepteurs ont ensuite démontré qu'une internalisation spécifique $\mathrm{m} / \mathrm{s} n^{\circ} 2$ vol. 10, féurier 94 branes plasmiques affecte les résidus sérine et tyrosine en proportion à peu près identique, le récepteur de l'insuline présent dans le compartiment endosomique est exclusivement phosphorylé sur les résidus tyrosine [17]. En outre, la concentration de récepteur phosphorylé est trois fois plus élevée dans l'endosome que dans la membrane plasmique, suggérant qu'il dissémine probablement le signal hormonal à l'intérieur de la cellule. A l'inverse, pour le récepteur du glucagon, son internalisation ne s'accompagne pas d'une co-internalisation des protéines régulatrices et catalytiques du système adénylyl cyclase, ce qui explique son incapacité à stimuler l'adénylyl cyclase et donc à promouvoir la production d'AMPc [16]. Ainsi, les récepteurs du glucagon retrouvés dans le compartiment endosomique sont insensibles à l'action des nucléotides guanyliques (comme le GTP) et reconnaissent le glucagon avec une faible affinité. Cela est la résultante du découplage entre les récepteurs du glucagon transportés dans l'endosome et les protéines-G qui restent à la surface cellulaire (figure 1). La mise en évidence d'une translocation des récepteurs du glucagon sous une forme inactive est un mécanisme classique de désensibilisation de type homologue des récepteurs couplés à l'adénylyl cyclase comme le récepteur $\beta$-adrénergique, qui, dans la plupart des types cellulaires, est internalisé sans son sytème de transduction. D'un point de vue général, seuls les récepteurs qui possèdent, par leur structure primaire, la possibilité d'engendrer des signaux biochimiques (récepteur de l'insuline et de l'EGF par exemple) auront leur activité fonctionnelle maintenue après leur internalisation [18].

glucagon accompagne celle de leur ligand respectif $[15,16]$. Cependant, une différence essentielle porte sur l'activité fonctionnelle des récepteurs de l'insuline et du glucagon internalisés (figure 1). Pour le récepteur de l'insuline, il a été établi que son autophosphorylation, ainsi que sa capacité à phosphoryler des substrats exogènes, sont maintenues dans le compartiment endosomique de l'hépatocyte [15]. Alors que l'autophosphorylation du récepteur de l'insuline présent dans les mem-

\section{L'endosome: site majeur de dégradation de l'insuline et du glucagon internalisés}

L'hypothèse d'une dégradation intraendosomique de l'insuline remonte aux travaux des années 1980 portant sur l'effet de la chloroquine, un agent acidotropique, sur 


\section{RÉFÉRENCES}

14. Jelinek LJ, Lok S, Rosenberg GB, et al. Expression cloning and signaling properties of the rat glucagon receptor. Science 1993 ; 259 : 1614-6.

15. Khan MN, Savoie S, Bergeron JJM, Posner BI. Characterization of rat liver endosomal fractions. In vivo activation of insulin-stimulable receptor kinase in these structures. J Biol Chem 1986 ; 261 : 8462-72.

16. Authier F, Desbuquois B, De Galle B. Ligand-mediated internalization of glucagon receptors in intact rat liver. Endocrinology 1992 ; 131 : 447-57.

17. Khan MN, Baquiran G, Brule C, Burgess J, Foster B, Bergeron IJM, Posner B. Internalization and activation of rat liver insulin receptor kinase in vivo. / Biol Chem 1989 ; 264: 12931-40.

18. Khan MN, Lai WH, Burgess IW, Posner BI, Bergeron IJM. Potential role of endosomes in transmembrane signaling. In : Bergeron IJM, Harris JR, eds. Subcellular biochemistry. endocytic components: identification and characterization. New York: Plenum Press, 1993: 237-68.

19. Hamel FG, Posner BI, Bergeron IJM, Frank BH, Duckworth WC. Isolation of insulin degradation products from endosomes derived from intact rat liver. I Biol Chem 1988; 263: 6703-8.

20. Doherty II II, Kay DG, Lai WH, Posner BI, Bergeron IJM. Selective degradation of insulin within rat liver endosomes / Cell Biol 1990; 1 10: 35-42.

21. Authier F, Desbuquois B. Degradation of glucagon in isolated liver endosomes. Biochem J 1991 ; 280 : 211-8.

22. Backer JM, Kahn CR, White MF. The dissociation and degradation of internalized insulin occur in the endosomes of rat hepatoma cells. J Biol Chem 1990; 265: 14828-35.

23. Authier F, Rachubinski RA, Posner BI, Bergeron IJM. Endosomal proteolysis of insulin by an acidic thiol-metalloprotease unrelated to insulin degrading enzyme (IDE). J Biol Chem 1994 (sous presse).

24. Duckworth WC. Insulin degradation : mechanisms, products and significance. Endocrinol Rev 1988; 9 : 319-45.

25. Shii K, Yokono K, Baba S, Roth RA Purification and characterization of insulindegrading enzyme from human erythrocytes. Diabetes 1986; $35: 675-83$.

26. Ebrahim A, Hamel FG, Bennett RG Duckworth WC. Identification of the metal associated with the insulin degrading enzyme. Biochem Biophys Res Commun 1991; 181: 1398-406.

27. Ogawa W, Shii K, Yonezawa K, Baba $\mathrm{S}$, Yokono $\mathrm{K}$. Affinity purification of insulin-degrading enzyme and its endogenous inhibitor from rat liver. J Biol Chem 1992 : $267: 1310-6$. l'endocytose hépatique de l'insuline. L'effet de cette substance injectée à des rats est d'augmenter l'accumulation de l'insuline dans le compartiment endosomique [9]. En outre, l'intégrité de l'insuline associée à ce compartiment est fortement augmentée chez ces animaux. Cet effet de la chloroquine, qui a été confirmé par la suite par des études similaires avec le glucagon [11], a été attribué à une diminution de la dégradation des ligands dans l'endosome et/ou de la dissociation des complexes ligand/récepteur, par une élévation du $\mathrm{pH}$ interne de ces vésicules.

Cette hypothèse a été ensuite confirmée par deux approches. La première a consisté à extraire des endosomes fraîchement préparés l'insuline et le glucagon internalisés dans ce compartiment et à analyser leur intégrité structurale par chromatographie liquide haute performance. Ainsi, des produits de dégradation de l'insuline ont été identifiés, avec des sites de clivage dans la chaîne A en position Leu ${ }^{13}-$ Tyr $^{14}$ et Tyr $^{14}-G n^{15}$ et dans la chaîne B en position $\mathrm{Ser}^{9}-\mathrm{His}^{10}$, Glu ${ }^{13}-\mathrm{Ala}^{14}$, Tyr ${ }^{16}-$ Leu $^{17}$, Phe ${ }^{25}-$ Tyr $^{26}$, His ${ }^{10}-$ Leu $^{11}$, Ala $^{14}-$ Leu $^{15}$ et Phe ${ }^{24}-$ Phe $^{25}$ [19], ainsi que dans la molécule de glucagon en position $\mathrm{Ser}^{2}-\mathrm{Gln}^{3}$, $\mathrm{Thr}^{5}-\mathrm{Phe}^{6}$ et $\mathrm{Phe}^{6}-\mathrm{Thr}^{7}$ (figure 2) [11].

La seconde approche a consisté à utiliser un système acellulaire d'endosomes isolés chargés in vivo en insuline et en glucagon iodés [20, 21]. Dans des conditions d'incubation isotonique, les endosomes maintiennent parfaitement leur capacité protéolytique intraluminale. Cela est confirmé par le fait que ces vésicules peuvent engendrer in vitro des produits de dégradation de l'insuline et du glucagon identiques à ceux retrouvés in vivo [21]. Ainsi, la dégradation est maximale à $\mathrm{pH}$ acide $(5,5$ pour l'insuline et 4 pour le glucagon) (figure 3A) démontrant que l'acidité du compartiment endosomique est requise pour une protéolyse des ligands. Une protéolyse des deux ligands peut cependant être observée à $\mathrm{pH}$ neutre si l'ATP, un substrat de la pompe à protons endosomique, est ajouté au milieu d'incubation (figure 3B). Parallèlement, l'ATP abaisse le $\mathrm{pH}$ interne des endosomes estimé par des mesures de fluorescence au moyen de l'acridine orange. L'effet de l'ATP est optimal en présence d'anions perméants (l'anion $\mathrm{Cl}$ étant le plus efficace) et est aboli par les ionophores carboxyliques (comme la monensine, figure $3 C$ ), par les inhibiteurs de l'ATPase à protons (comme la $\mathrm{N}$-éthylmaléimide) et par les agents acidotropiques (comme la chloroquine ou la dansylcadavérine). Cependant, les inhibiteurs des $\mathrm{Na}^{+}, \mathrm{K}^{+}-\mathrm{ATPases}$ de la membrane plasmique (vanadate et ouabaïne) n'interfèrent pas avec l'effet de l'ATP [20, 21]. L'ensemble de ces résultats indiquent que la capacité de l'ATP d'affecter la dégradation de l'insuline et du glucagon dans les endosomes est liée exclusivement à sa capacité d'acidifier ces organites. L'observation que la dégradation intraendosomique de ces deux hormones est maximale à $\mathrm{pH}$ acide $(\mathrm{pH}$ auquel l'affinité des hormones pour leur récepteur est presque nulle) montre que la dissociation des complexes hormone/récepteur est requise pour la dégradation des ligands, le ligand dissocié étant le substrat physiologique des protéases luminales (figure 4).

La sensibilité de la dégradation intraendosomique de l'insuline à différents inhibiteurs de protéases et différentes bases faibles [20] est assez comparable à celle observée pour le glucagon [21]. Ainsi, la dégradation est-elle inhibée principalement par les chélateurs de métaux, par les réactifs des groupements thiols et par la bacitracine. Aucun effet n'est cependant observé avec les inhibiteurs des protéases à sérine. Ces résultats militent en faveur de l'implication de plusieurs thiol-endopeptidases et métalloendopeptidases dont l'activité optimale se manifeste à $\mathrm{pH}$ acide dans le processus d'inactivation endosomique de l'insuline et du glucagon.

La spécificité de l'insulinase endosomique envers l'insuline est démontrée par le fait que cette protéase ne semble pas exprimée dans des cellules insensibles à l'insuline [22] Ainsi, alors qu'une dégradation endosomique de l'insuline est aisément détectable dans les cellules Fao qui expriment naturellement le récepteur de l'insuline, seule une dégradation lysosomiale de l'insuline 


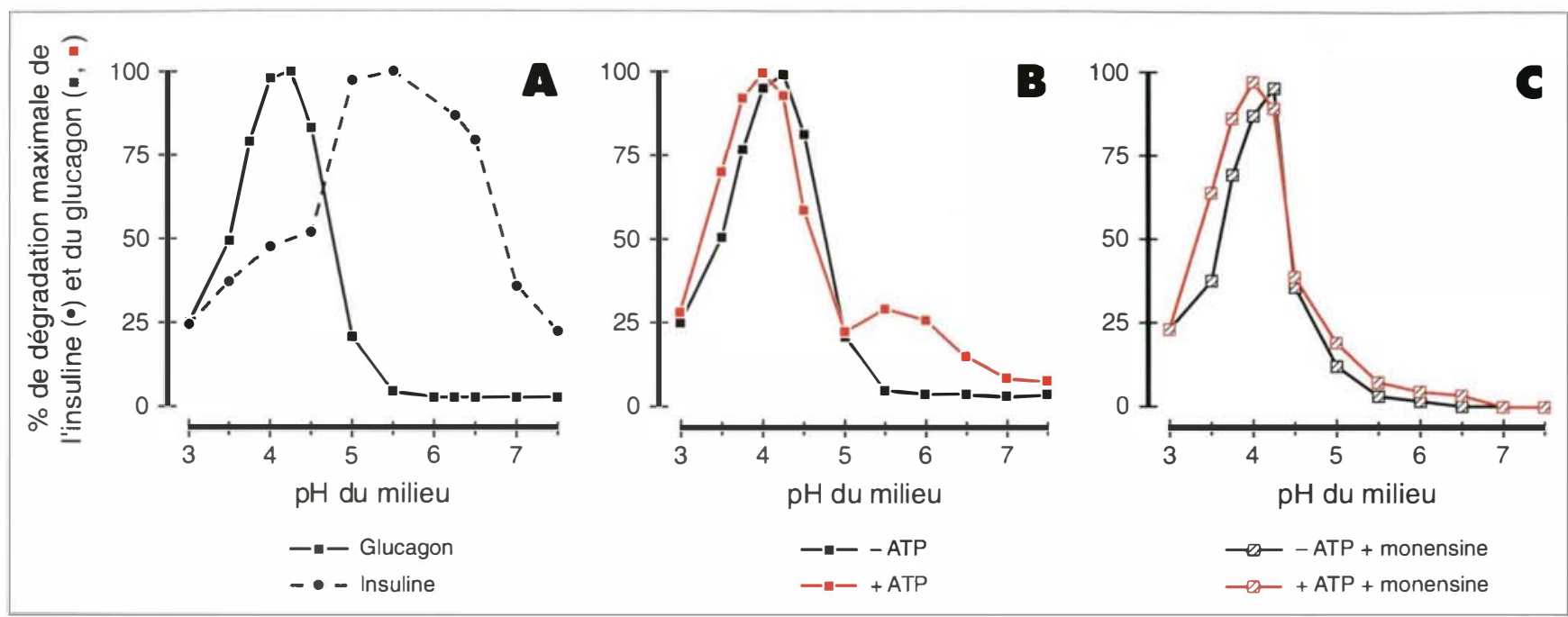

Figure 3. Effets du pH et de I'ATP sur la dégradation intraendosomique de l'insuline et du glucagon dans un système acellulaire d'endosomes isolés. Les endosomes sont chargés in vivo en insuline iodée (A) ou en glucagon iodé (A, B et $\boldsymbol{C})$. Puis, les vésicules d'endocytose sont incubées à différents $\mathrm{pH}(\mathbf{A})$, à différents pH en présence d'ATP $(B)$, et à différents $\mathrm{pH}$ en présence d'ATP et de monensine (un ionophore carboxylique qui abolit le gradient de protons transmembranaire) (C). Un effet similaire de la monensine peut être obtenu avec l'addition $d^{\prime} u n$ inhibiteur de $\mathrm{I}^{\prime} \mathrm{H}^{+}$-ATPase (comme la $\mathrm{N}$-éthylmaléimide) ou d'une base faible (comme la chloroquine et la dansyl-cadavérine). La dégradation est appréciée par une quantification du taux de radioactivité soluble dans l'acide trichloroacétique.

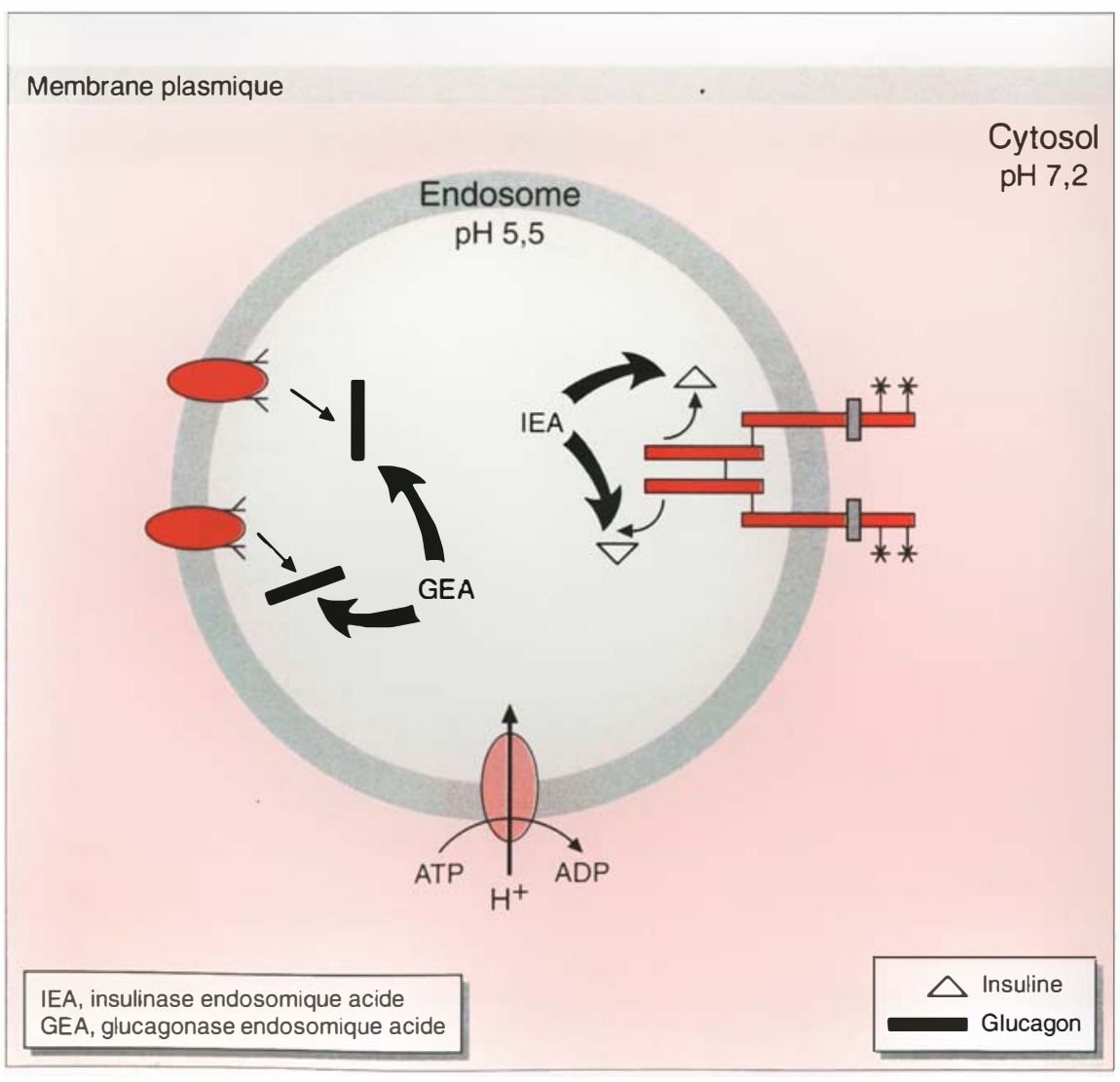

$\mathrm{m} / \mathrm{s} n^{\circ} 2$ vol. 10 , fériner 94
Figure 4. Dégradation intraendosomique de l'insuline et du glucagon dans l'hépatocyte. Les complexes hormone/récepteur sont dissociés par l'acidité du $\mathrm{pH}$ qui est entretenue par l'activité de la pompe à protons dépendante de I'ATP. L'insuline et le glucagon libres dans la partie luminale de l'endosome sont ensuite protéolysés par l'action de différentes thiol-endopeptidases dont l'activité est optimale à $\mathrm{pH}$ acide. Les produits de dégradation diffuseront alors hors de l'endosome dans le compartiment cytoplasmique. 


\section{RÉFÉRENCES}

28. Baumeister H, Müller D, Rehbein M, Richter D. The rat insulin-degrading enzyme: molecular cloning and characterization of tissue-specific transcripts. FEBS Lett 1993; 317 : 250-4.

29. Aftholter JA, Hsieh C.L, Francke U, Roth RA. Insulin-degrading enzyme : stable expression of the human complementary DNA, characterization of its protein product, and chromosomal mapping of the human and mouse gene. Mol Endocrino $1990 ; 4: 1125-35$.

30. Kuo WL, Gehm BD, Rosner MR. Cloning and expression of the cDNA for a Drosophila insulin-degrading enzyme. $\mathrm{Mol}$ Endocrinol 1990; 4:1580-91.

31. Ding L, Becker AB, Suzuki A, Roth RA. Comparison of the enzymatic and biochemical properties of human insulindegrading enzyme and Escherichia coli protease III. J Biol Chem 1992 ; 267 : 2414-20.

32. Kole HK, Smith DR, Lenard J. Characterization and partial purification of an insulinase from Neurospora crassa. Arch Bio chem Biophys 1992; 297 : 199-204.

33. Gould SJ, Keller GA, Hosken N, W'ilkinson J, Subramini S. A conserved tripeptide sorts proteins to peroxisomes. I Cell Biol 1989; 108: 1657-64.

34. De Duve. Exploring cells with a centrifuge. Science 1975 ; 189 : 186-94.

35. Diment S, Leech MS, Stahl PD. Cathepsin D is membrane-associated in macrophage endosomes. I Biol Chem 1989; 263 : 6901-7.

36. Diment S, Martin KJ, Stahl PD. Cleavage of parathyroid hormone in macrophage endosomes illustrates a novel pathway for intracellular processing of proteins. J Biol Chem 1989; 264: 13403-6.

37. Blum JS, Diaz R, Mayorga LS, Stahl PD. Reconstitution of endosomal transport and proteolysis. In : Bergeron IIM, Harris JR, eds. Subcellular biochemisly'. Endocylic com ponenls: idenlificalion and characlerizalion. New York: Plenum Press, 1993 : 69-93.

38. Renfrew C.A, Hubbard AL. Sequential processing of epidermal growth factor in early and late endosomes of rat liver. I Biol Chem 1991; 266 : 4348-56.

39. Standl E, Kolb HJ. Insulin degrading enzyme activity and insulin binding of erythrocytes in normal subjects and type 2 (non-insulin-dependent) diabetic patients. Diabelologia 1984; 27 : 17-22.

40. Maberly GF, W'ait GA, Kilpatrick JA, $e$ al. Evidence for insulin degradation by muscle and fat tissue in an insulin resistant diabetic patient. Diabelologia $1982 ; 23$ : 333-6.

41. Blazar BR, W'hitley C.B, Kitabchi AE, et al. In vivo chloroquine-induced inhibition of insulin degradation in a diabetic patient with severe insulin resistance. I ia betes $1984 ; 33: 1133-7$.

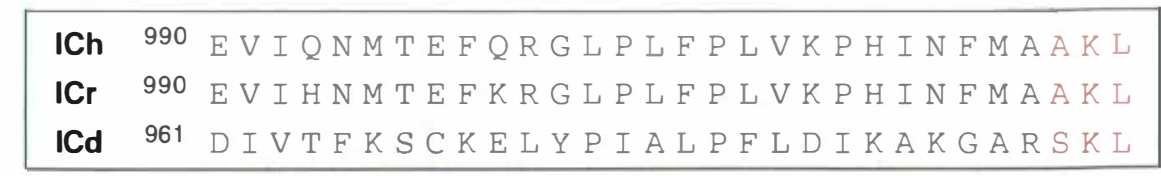

Figure 5. Séquence carboxyterminale de I'insulinase cytosolique humaine (ICh), de rat (ICr) et de drosophile (ICd). Le tripeptide AVSKL en position carboxyterminale est une condition suffisante pour le ciblage des protéines dans le peroxysome [33].

est détectée dans les cellules $\mathrm{CHO}$ transfectées par l'ADNc du récepteur de l'insuline [22]. Récemment, la purification partielle de l'insulinase endosomique a mis en évidence une protéine majeure de $80 \mathrm{kDa}$ qui se lie à l'insuline à $\mathrm{pH}$ acide et qui représente donc une protéase candidate potentielle [23]. Cette protéine endosomique est distincte au point de vue structural et immunologique de l'insulinase cytosolique, une thiolmétalloendopeptidase dont l'activité optimale se manifeste à $\mathrm{pH}$ neutre et de localisation présumée cytoplasmique [23].

\section{L'insulinase cytosolique: une nouvelle protéase peroxysomiale}

Une approche biochimique a conduit à l'identification, en 1972, d'une protéase localisée dans une fraction cytosolique de foie de rat et capable de dégrader l'insuline et le glucagon in vitro. Du fait de son affinité remarquable pour l'insuline $\left(\mathrm{Km}=10^{-x} \mathrm{M}\right)$, elle fut dénommée insulin degrading enzyme, insulin protease ou "Insulinase", et fut, de ce fait, présentée comme l'enzyme clé du métabolisme intracellulaire de l'insuline et du glucagon [24]. Ses principales propriétés biochimiques incluent: un poids moléculaire de 300 kDa, évalué par filtration sur gel; une sous-unité de 110 kDa, estimée par électrophorèse sur gel de polyacrylamide en conditions dénaturantes et réductrices; un $\mathrm{pH}$ optimal de 7 ; et l'inhibition de son activité par les réactifs des groupements thiols, par les chélateurs de métaux et par la bacitracine, démontrant l'appartenance de cette enzyme à la classe des thiol-métalloprotéases [25]. La détection rapide de cette protéase dans une fraction subcellulaire est possible en utilisant une technique de pontage covalent avec l'insuline iodée, suivie d'une analyse des complexes covalents [ $\left.{ }^{125} \mathrm{I}\right]$ insuline/insulinase cytosolique par électrophorèse sur gel de polyacrylamide. Ainsi, l'insulinase cytosolique a été identifiée dans un grand nombre d'autres tissus - principalement le cerveau, le muscle et le rein et dans un grand nombre de types cellulaires dont les adipocytes, les fibroblastes, et les cellules placentaires et pancréatiques [24]. Le nombre de substrats de l'insulinase cytosolique s'est récemment élargi avec la démonstration d'une dégradation in vitro par cette enzyme des facteurs de croissance (IGFI, IGFII et TGF $\alpha$ ) et du facteur natriurétique atrial (ANF) avec une affinité variable selon le substrat: $\mathrm{ANF}=$ TGF $\alpha>$ insuline $>$ IGF-I $>$ IGF-II $>$ glucagon [4]. L'identification des sites de clivage peptidique des substrats précédemment cités a montré une absence de spécificité au niveau des liaisons peptidiques clivées, suggérant que l'enzyme pourrait reconnaître davantage une structure secondaire (la molécule elle-même) qu'une séquence particulière d'acides aminés. Cependant, il semble que les clivages peptidiques s'effectuent préférentiellement à proximité des acides aminés hydrophobes de type leucine et tyrosine. L'activité de l'insulinase cytosolique semble influencée par certains facteurs puisque, par exemple, l'insulinase cytosolique requiert des cations divalents 
Tableau I

COMPARAISON DES PROPRIÉTÉS DE L'INSULINASE CYTOSOLIQUE NEUTRE (IC) ET DE L'INSULINASE ENDOSOMIQUE ACIDE DE L'HÉPATOCTYE DE RAT

\begin{tabular}{|c|c|c|}
\hline & $\begin{array}{c}\text { Insulinase cytosolique } \\
\text { neutre }\end{array}$ & $\begin{array}{l}\text { Insulinase endosomique } \\
\text { acide }\end{array}$ \\
\hline Masse moléculaire (kDa) & 110 & $80 ?$ \\
\hline Localisation subcellulaire & peroxysome/cytosol & endosome \\
\hline pH d'activité optimale & $7-8$ & $4,0-5,5$ \\
\hline $\begin{array}{l}\text { Inhibition de l'activité catalytique par } \\
\text { - réactifs des groupements thiols } \\
\text { - chélateurs de métaux } \\
\text { - bacitracine }\end{array}$ & $\begin{array}{l}+ \\
+ \\
+\end{array}$ & $\begin{array}{l}+ \\
+ \\
+\end{array}$ \\
\hline $\begin{array}{l}\text { Pontage covalent à pH neutre avec } \\
-[125] \text { insuline } \\
-[125] \text { glucagon }\end{array}$ & $\begin{array}{l}+ \\
+\end{array}$ & - \\
\hline $\begin{array}{l}\text { pH optimal de liaison sur colonne } \\
\text { d'affinité insuline-agarose }\end{array}$ & $6-7$ & 5 \\
\hline $\begin{array}{l}\text { Spécificité de substrat } \\
\text { - insuline } \\
\text { - glucagon }\end{array}$ & 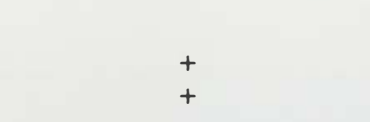 & + \\
\hline $\begin{array}{l}\text { Liaisons peptidiques clivées dans } \\
\text { la molécule d'insuline } \\
\text { - Chaîne A } \\
\text { - Chaîne B }\end{array}$ & $\begin{array}{llll}9-10 & 10-11 \quad \stackrel{13-14}{+} 14-15 \\
16-17 & 24-25 & 25-26\end{array}$ & $16-17 \quad 24-25 \quad 25-26$ \\
\hline $\begin{array}{l}\text { Reconnaissance immunologique } \\
\text { - anticorps monoclonal 9B12 anti-IC } \\
\text { - anticorps polyclonal 2BS anti-IC }\end{array}$ & + & - \\
\hline
\end{tabular}

La localisation subcellulaire, la spécificité de substrat, et les propriétés structurales, catalytiques et antigéniques de l'insulinase cytosolique neutre sont comparées à celles de l'insulinase endosomique acide. Pour les clivages peptidiques dans la molécule d'insuline, les nombres indiquent la position des acides aminés encadrant les liaisons peptidiques clivées consécutivement à l'action de l'une ou de l'autre des deux endopeptidases.

pour son activité enzymatique. Des études récentes indiquent que le zinc et le manganèse sont associés physiologiquement à l'insulinase cytosolique avec une concentration dix fois plus élevée pour le zinc que pour le manganèse [26]. L'inhibiteur endogène de l'insulinase cytosolique est aussi un facteur de régulation important pour l'activité de cette enzyme. Cet inhibiteur de 14 $\mathrm{kDa}$, récemment purifié à partir d'une fraction cytosolique de foie de rat, est de type compétitif. Il est en partie associé physiologiquement à l'insulinase cytosolique et empêche l'accessibilité des substrats au site actif de cette protéase [27].

Purifiée à homogénéité à partir d'érythrocytes humains [25] et de $\mathrm{m} / \mathrm{s} n^{\circ} 2$ vol. 10, feurier 94 cerveau de rat [28], l'insulinase cytosolique a vu son $\mathrm{ADNc}$ cloné chez ces deux espèces, et la détermination de la séquence primaire a révélé $95 \%$ d'homologie en acides aminés [28, 29]. L'insulinase cytosolique humaine partage $48 \%$ d'analogie de séquence avec une protéase identifiée chez la mouche Drosophila melanogaster [30] et $26 \%$ d'analogie avec une protéase périplasmique identifiée chez la bactérie Escherichia coli et appelée «protéase III ou $\mathrm{Pi}$ »[31]. Trois régions de forte similitude comportant $57 \%$ à $80 \%$ d'acides aminés identiques ont été identifiées chez ces quatre espèces, indiquant que la structure primaire de l'insulinase cytosolique est hautement conservée au cours de l'évolution. Cette iden- tité structurale est associée à une conservation remarquable des propriétés enzymatiques de l'insulinase cytosolique. En effet, la masse moléculaire, le $\mathrm{pH}$ optimal, les cations bivalents stimulant l'activité de l'enzyme, les inhibiteurs, la spécificité de substrat ainsi que les liaisons peptidiques clivées au niveau de la molécule d'insuline sont très semblables [4]. Récemment, une protéase dégradant l'insuline et partageant les mêmes propriétés enzymatiques que l'insulinase cytosolique a été identifiée chez le champignon Neurospora crassa, élargissant ainsi le spectre phylogénétique de cette protéase [32].

Depuis quelques années, l'implication de l'insulinase cytosolique dans la dégradation physiologique de 
l'insuline et du glucagon est fortement controversée, et ce pour de multiples raisons: (1) pour son $\mathrm{pH}$ d'activité optimale neutre, alors que l'on sait que la dégradation intracellulaire de l'insuline et du glucagon est maximale à $\mathrm{pH}$ acide ; (2) pour sa localisation subcellulaire présumée cytosolique qui ne coïncide pas avec celle des hormones polypeptidiques internalisées, qui est endosomique; (3) pour la découverte récente d'un grand nombre de substrats, autres que l'insuline et le glucagon, et qui ont pour l'insulinase cytosolique une affinité égale, voire supérieure, à celle de ces deux hormones; et enfin (4) pour sa présence dans des systèmes bactériens qui ne sont pas sensibles à l'insuline (comme Escherichia coli). Dans une étude publiée en 1993 [28], la comparaison des parties carboxyterminales de l'insulinase cytosolique humaine, de rat et de drosophile a révélé la séquence consensus A/SKI. en position C-terminale (figure 5). Ce tripeptide est impliqué dans le ciblage spécifique des protéines vers le peroxysome [33]. Dès lors, l'analyse de la distribution subcellulaire de l'insulinase cytosolique dans l'hépatocyte de rat a été entreprise et celle-ci a révélé que cette enzyme n'était pas détectée par les techniques de pontage covalent et d'immunodétection classiques dans des endosomes hautement purifiés, mais que, cependant, elle était bien localisée dans la fraction cytosolique du foie de rat ainsi que dans des peroxysomes (Tableau I) [23]. Considéré dès 1975 par C. de Duve comme un organite fragile [34], le peroxysome, préparé par fractionnement subcellulaire, libère bon nombre de ses constituants intraluminaux (comme $35 \%$ de sa catalase) dans la fraction cytosolique au cours des étapes de fractionnement subcellulaire. Cette fragilité peroxysomiale pourrait donc être à l'origine d'une présence artéfactuelle de l'insulinase cytosolique dans la fraction cytosolique soluble. Cependant, une double localisation subcellulaire (cytoplasmique et peroxysomiale) de l'enzyme est aussi envisageable [23]. Malgré un rôle encore mal connu, l'insulinase cytosolique semble impliquée dans deux processus physiolo- giques importants: la différenciation des myoblastes L6, et la croissance et le développement du rat et de la drosophile. Dans ces deux espèces, une augmentation graduelle de l'expression de cette enzyme a lieu du stade embryonnaire au stade adulte [4].

\section{Autres protéolyses endosomiques identifiées}

D'autres événements protéolytiques impliquant des hormones, des facteurs de croissance, des toxines et des antigènes étrangers ont été identifiés dans le compartiment endosomique de types cellulaires variés (Tableau II). Les protéases responsables n'ont pas toutes été caractérisées, excepté la cathepsine D, purifiée à partir de l'endosome de macrophage alvéolaire de lapin [35]. Dans ce type cellulaire, cette protéase est responsable de la dégradation de la chaîne $\mathrm{A}$ de la ricine, de la sérum albumine mannosylée et de l'hormone parathyroïdienne (PTH). Dans l'hépatocyte, elle pourrait être impliquée dans la dégradation endosomique des apolipoprotéines B-100. Les clivages peptidiques affectant la séquence de la PTH libèrent le fragment 1-34 qui est un produit de dégradation possédant une activité biologique et qui est recyclé à la surface cellulaire [36]. L'étude de la biosynthèse de la cathepsine $\mathrm{D}$ a montré que cette protéase est synthétisée sous la forme d'un précurseur inactif (procathepsine D) de 53 kDa qui, à partir de l'appareil de Golgi, est ciblé vers l'endosome sous une forme associée à la membrane. Dans l'endosome, un processus de maturation convertit le précurseur inactif membranaire en une protéase active soluble de $46 \mathrm{kDa}$ qui constitue l'unique forme retrouvée dans les lysosomes [37]. Ia cathepsine $\mathrm{B}$ a également été détectée par des méthodes biochimiques et morphologiques dans l'endosome de divers types cellulaires, alors que la leucine aminopeptidase n'a été identifiée que dans le compartiment endosomique des cellules HeLa [37]. Enfin, une carboxypeptidase et une activité de type trypsine sont responsables de l'élimination séquentielle de plusieurs acides ami- nés de la partie carboxyterminale de l'EGF dans les endosomes précoces et tardifs des hépatocytes et des fibroblastes [4, 38]. Les protéases endosomiques, responsables de la dégradation des triglycérides de type VIDL dans l'hépatocyte et des antigènes étrangers dans les cellules immunitaires, n'ont cependant pas été caractérisées [37]. D'autres modifications structurales de molécules internalisées dans l'endosome ont été décrites et concernent principalement la réduction des ponts disulfures des toxines bactériennes comme les toxines de la diphtérie et du choléra, et des toxines végétales telles que la ricine [37]. Les enzymes impliquées dans ces mécanismes de réduction n'ont pas encore été caractérisées.

\section{Conclusion}

L'endosome possède donc, en plus de ses fonctions classiques dans le trafic et le tri cellulaires de diverses molécules, des capacités protéolytiques qui en font, avec le lysosome, un organite majeur de la dégradation intracellulaire des molécules internalisées. Cependant, alors que la protéolyse lysosomiale semble être peu spécifique, la dégradation endosomique dépasse largement le cadre d'une inactivation passive des molécules endocytées. Bien au contraire, la protéolyse endosomique fait partie intégrante d'un grand nombre de processus biologiques, d'une part, par sa capacité à engendrer des produits de dégradation biologiquement "actifs" qui seront ensuite recyclés vers la membrane plasmique, et d'autre part, par son implication dans la protéolyse et la présentation des antigènes étrangers aux cellules immunitaires. En outre, par sa capacité à dégrader sélectivement les hormones et les facteurs de croissance, l'endosome est capable de moduler la dissémination intracellulaire d'un signal hormonal [18].

L'importance physiopathologique de la dégradation intracellulaire de l'insuline est illustrée par le fait qu'une augmentation des activités protéolytiques dégradant l'insuline a été observée dans des érythrocytes de sujets atteints de diabète de type 2 [39] et dans des biopsies de 
Tableau II

\section{PROTÉASES IDENTIFIÉES DANS LE COMPARTIMENT ENDOSOMIQUE DE DIVERS TYPES CELLULAIRES}

\begin{tabular}{|lcc|}
\hline Protéases & Types celluiaires & $\begin{array}{c}\text { Substrats } \\
\text { physiologiques }\end{array}$ \\
\hline $\begin{array}{l}\text { Insulinase endosomique acide } \\
\text { acide }\end{array}$ & Hépatocyte & Insuline \\
Cathepsine D & Hépatocyte & Glucagon \\
& Macrophage & $\begin{array}{c}\text { Sérum } \\
\text { albumine-mannosylée } \\
\text { PTH-(1-84) }\end{array}$ \\
& Chaîne A de la ricine & Vitellogénine \\
Cathepsine B & Hépatocyte & Apolipoprotéine B-100? \\
& Fibroblaste & EGF \\
Activité de type trypsine & HepG2 & $?$ \\
Leucine aminopeptidase & Hépatocyte & EGF \\
\hline
\end{tabular}

muscle et de tissu adipeux de sujets atteints de diabète de type 1 [40]. En outre, la restauration d'une dégradation normale de l'insuline chez un patient diabétique présentant une résistance à l'insuline a pu être obtenue par une thérapie à la chloroquine [41], suggérant qu'une augmentation de l'expression d'une insulinase acide pourrait être en cause dans certains diabètes. Il est donc important que les investigations futures portent sur la structure et l'expression au niveau moléculaire des protéases endosomiques impliquées dans la dégradation des hormones et des facteurs de croissance. Ces recherches présenteront deux axes complémentaires d'investigation: le premier purement fondamental, visant à déterminer la signification physiologique ainsi que les systèmes de ciblage et de rétention de ces enzymes dans le compartiment endosomique; le second, plus appliqué, qui vise à apprécier l'implication de ces processus de dégradation dans certaines maladies qui s'accompagnent d'une protéolyse intracellulaire accrue ou diminuée pour certaines molécules

\section{TIRÉS A PART}

J.J.M. Bergeron

\section{Summary}

Hepatic endosomes are the major physiological locus of insulin and glucagon degradation in vivo

The major hormones regulating glucose homeostasis are insulin and glucagon. The islets of Langerhans in the pancreas are anatomically positioned to deliver these hormones to the portal circulation of the liver. Insulin and glucagon secreted from the pancreas are extracted from the portal circulation to the extent of $>45 \%$ in a single pass through the liver. Liver clearance of these pancreatic hormones is effected mainly by a receptor-mediated mechanism. After binding to the hepatic plasmalemma, hormone/receptor complexes are rapidly internalized into components of the endosomal apparatus, inside which a progressive degradation of internalized hormones has been demonstrated. ATP-dependent acidification is required for optimal degradation of insulin and glucagon within endosomes by distinct proteinases whose nature remains undefined. It has been recently shown that the insulin and glucagon proteinases within endosomes display an acidic $\mathrm{pH}$ optimum and are unrelated to insulin degrading enzyme (IDE), a cytosolic thiol-metalloprotease proposed previously to be the main cellular clearance mechanism. This latter enzyme has been observed in peroxisomes and indeed possesses a classical A/SKL peroxisomal targeting motif at its extreme carboxy termini and is unlikely to be relevant to hormone degradation in vivo. Since ligand degradation is an important process for control of hormone action, the endosomal hormone degrading proteinases that we have identified may be clinically relevant and may represent important potential targets for pharmaceutical agents which control hormone action. 